\section{Structure of $d$ (GCGAAAGC) (hexagonal form): a base-intercalated duplex as a stable structure. Erratum}

Tomoko Sunami, ${ }^{\text {a Jiro Kondo, }}{ }^{\text {a }}$ Ichiro Hirao, ${ }^{\mathrm{b}, \mathrm{c}}$ Kimitsuna Watanabe, ${ }^{d}$ Kin-ichiro Miura ${ }^{e}$ and Akio Takénaka ${ }^{a} *$

${ }^{a}$ Graduate School of Bioscience and Biotechnology, Tokyo Institute of Technology, Yokohama 226-8501, Japan, 'bIKEN GSC, Wako-shi, Saitama 351-0198, Japan, ${ }^{\mathbf{c}}$ Research Center for Advanced Science and Technology, University of Tokyo, Tokyo 153-8904, Japan, ${ }^{\mathbf{d} G r a d u a t e}$ School of Engineering, University of Tokyo, Tokyo 1138656, Japan, and ${ }^{\mathbf{e}}$ Faculty of Science, Gakushuin University, Tokyo 171-8588,

Japan. Correspondence e-mail: atakenak@bio.titech.ac.jp

In the paper by Sunami et al. [(2004), Acta Cryst. D60, 90-96] an incorrect version of Table 2 was published. The correct version is given here.

\section{References}

Lu, X.-J. \& Olson, W. K. (2003). Nucleic Acids. Res. 31, 5108-5121.

Shepard, W., Cruse, W. B., Fourme, R., de La Fortelle, E. \& Prangé, T. (1998). Structure, 6, 849-861.

Sunami, T., Kondo, J., Hirao, I., Watanabe, K., Miura, K. \& Takenaka, A. (2004). Acta Cryst. D60, 90-96
Table 2

Some helical parameters and sugar puckers of the base-intercalated duplexes.

Inclin, Prop, Buckl and Open represent inclination, propeller twist, buckle and opening angles (see Lu \& Olson, 2003). The $\mathrm{C}_{2}$ residue is iodinated in $9 \mathrm{hmt}-\mathrm{I}$ and brominated in $9 \mathrm{hmt}-\mathrm{Br}$. For each base pair, the upper line is for $9 \mathrm{hmt}-\mathrm{I}$, the middle line for $8 \mathrm{hmt}-\mathrm{h}$ and the lower line for 9hmt-Br (Shepard et al., 1998). The corresponding values for the remaining half of the duplex are omitted owing to crystallographic symmetry.

(a) Helical parameters.

\begin{tabular}{lrrrrrrrr}
\hline Base pair & Inclin & Tip & Twist & $\mathrm{dz}$ & Prop & Buckl & Open & $\mathrm{C1}^{\prime} \cdots \mathrm{C}^{\prime}$ \\
\hline $\mathrm{G}_{1}: \mathrm{C}_{8}^{*}$ & 1 & -6 & 26 & 3.1 & 6 & -2 & -3 & 10.7 \\
& -2 & -8 & 28 & 3.0 & 9 & 0 & 3 & 10.5 \\
$\mathrm{C}_{2}: \mathrm{G}_{7}^{*}$ & 2 & 3 & 31 & 3.2 & 6 & -1 & 1 & 10.5 \\
& 6 & 0 & 55 & 3.3 & 10 & 13 & 0 & 10.5 \\
& 10 & 3 & 52 & 3.2 & 4 & 16 & 1 & 10.5 \\
$\mathrm{G}_{3}: \mathrm{A}_{6}^{*}$ & 10 & 0 & 52 & 3.4 & 8 & 9 & -1 & 10.6 \\
& $\dagger$ & $\dagger$ & $\dagger$ & $\dagger$ & -12 & 29 & 9 & 8.3 \\
& $\dagger$ & $\dagger$ & $\dagger$ & $\dagger$ & -4 & 31 & 8 & 8.4 \\
A form & $\dagger$ & $\dagger$ & $\dagger$ & $\dagger$ & -15 & 25 & 10 & 8.5 \\
B form & -5 & 0 & 33 & 2.3 & 12 & 0 & -2 & 10.7 \\
\hline
\end{tabular}

(b) Sugar puckers.

\begin{tabular}{|c|c|c|c|}
\hline Nucleotide & Pucker, 9hmt-I & Pucker, 8hmt-h & Pucker, 9hmt-Br \\
\hline $\mathrm{G}_{1}$ & $\mathrm{C}^{\prime}$-exo & $\mathrm{C} 3^{\prime}$-exo & $\mathrm{C}^{\prime}$-exo \\
\hline $\mathrm{C}_{2}$ & $\mathrm{C1}^{\prime}$-exo & $\mathrm{C1}^{\prime}$-exo & $\mathrm{C1}^{\prime}$-exo \\
\hline $\mathrm{G}_{3}$ & $\mathrm{C} 2^{\prime}$-endo & $\mathrm{C} 2^{\prime}$-endo & $\mathrm{C} 2^{\prime}$-endo \\
\hline $\mathrm{A}_{4}$ & $\mathrm{C}^{\prime}$-endo & $\mathrm{C}^{\prime}$-endo & $\mathrm{C}^{\prime}$-exo \\
\hline $\mathrm{A}_{5}$ & $\mathrm{C1}^{\prime}$-exo & $\mathrm{C1}^{\prime}$-exo & $\mathrm{C1}^{\prime}$-exo \\
\hline$A_{6}$ & $\mathrm{C} 2^{\prime}$-endo & $\mathrm{C} 2^{\prime}$-endo & $\mathrm{C} 2$ '-endo \\
\hline $\mathrm{G}_{7}$ & $\mathrm{C1}^{\prime}$-exo & $\mathrm{C1}^{\prime}$-exo & $\mathrm{C} 2$-endo \\
\hline $\mathrm{C}_{8}$ & $\mathrm{C} 2$ '-endo & $\mathrm{C} 4^{\prime}-$ exo & $\mathrm{C} 3^{\prime}$-exo \\
\hline $\mathrm{T}_{9}$ & $\ddagger$ & + & $\ddagger$ \\
\hline A form & $\mathrm{C}^{\prime}$-endo & $\mathrm{C} 3^{\prime}$-endo & C3'-endo \\
\hline B form & $\mathrm{C} 2$ '-endo & $\mathrm{C} 2^{\prime}$-endo & $\mathrm{C} 2^{\prime}$-endo \\
\hline
\end{tabular}

$\dagger \mathrm{A}_{4}$ and $\mathrm{A}_{5}$ are not paired with any bases. $\$ \mathrm{~T}_{9}$ is disordered. 